Noname manuscript No.

(will be inserted by the editor)

\title{
Multi-functional, optofluidic, in-plane, bi-concave lens: \\ tuning light beam from focused to divergent
}

\author{
Chaolong Song · Nam-Trung Nguyen • Yit \\ Fatt Yap · Trung-Dung Luong · Anand
}

Krishna Asundi

\begin{abstract}
The miniaturization and integration of in-plane micro lenses into microfluidic networks for improving fluorescence detection has been widely investigated recently. This paper describes the design and demonstration of an optofluidic in-plane bi-concave lens to perform both light focusing and diverging. The concave lens is hydrodynamically formed in a rectangular chamber with a liquid core liquid cladding $\left(\mathrm{L}^{2}\right)$ configuration. In the focusing mode, an auxiliary cladding stream is introduced to sandwich the $\mathrm{L}^{2}$ configuration for protecting the light rays from scattering at the rough chamber wall. In the diverging mode, the auxiliary cladding liquid changes its role from avoiding light-scattering to being the low-refractive-index cladding of the lens. The focal length in the focusing mode and the divergent angle of light beam in
\end{abstract}

\section{N. T. Nguyen}

School of Mechanical and Aerospace Engineering

Nanyang Technological University

50 Nanyang Avenue, Singapore, Singapore 639798

Tel.: $+65-6790-4457$

Fax: +65-6792-4062

E-mail: mntnguyen@ntu.edu.sg 
the diverging mode can be tuned by adjusting the flow rate ratio between core and cladding streams.

Keywords Micro optofluidics, liquid lens, bi-concave lens, beam focusing, beam diverging, laser sheet

\section{Introduction}

Fluorescence has been widely used in biochemistry and analytical chemistry as a nondestructive means of tracking or analysis of bio-molecules or chemicals. Traditionally, the excitation of fluorescence is introduced by employing an external mercury lamp with a filter set or a laser with a specific wavelength. Recently, the emergence of microfluidics has led to the realization of on-chip biological/chemical assays. Fluorescence excitation and detection have also been integrated into a chip to make the whole system cost-effective and portable [1-5]. To improve the performance of the fluorescence excitation and modify the light properties, a single solid-interface lens was integrated into microfluidic network [6]. A system of lenses was used to correct the optical aberration of the single lens [7]. However, the drawback of the solid-based lens is the roughness of interface, which can cause strong light scattering. Following this concept of in-plane light modulation, a number of tuneable configurations have been demonstrated, including the two-fluid controlled cylindrical microlens [8], liquid core liquid cladding $\left(\mathrm{L}^{2}\right)$ optofluidic lenses [9-12], and pressure controlled air-liquid-interface lens [13]. These in-plane lenses can dynamically modify the light introduced from an optical fibre. Moreover, their $\mathrm{L}^{2}$ configuration has an atomically smooth interface as opposed to the rough interface of solid based lenses. Currently, most of the in-plane light modifications only involve a tuneable light that is focused along the optical axis. 
A gradient-refractive-index lens has been reported to swing a focused light beam [14].

A liquid-core liquid-cladding prism has been proposed to continuously deflect a light beam [15]. These two works provide different ways of modifying the in-plane light, by which the area out of the optical axis can also be selectively illuminated, thus exciting the local fluorescence dye.

This paper describes the design and demonstration of an optofluidic bi-concave lens that can perform both light focusing and diverging. In traditional optics, a concave lens is fabricated with glass, which has a refractive index (RI) higher than that of surrounding medium, and thus cannot be used to focus light. The properties of an optofluidic component can be changed simply by replacing the liquid inside the component[16]. In the focusing mode of our optofluidic concave lens, a liquid with low refractive index is used as the core stream and a liquid with higher refractive index serves as the cladding stream, Figure 1 (a). It is found that for a $\mathrm{L}^{2}$ lens system the mis-match of refractive index between cladding liquid and PDMS brings about light scattering [10,12,17]. In this work, we introduce an auxiliary cladding liquid with a very low flow rate and a refractive index matching PDMS to prevent the light beam from scattering. Since the auxiliary streams are thin and has matching reflective index, their effect on light focusing is negligible. The light emitted from an optical fibre can be well focused and the focal length can be tuned by adjusting the flow rate ratio between core and cladding streams. If the core inlet is blocked, the liquid from cladding inlets will converge into a new core stream and the liquid from the auxiliary cladding inlet will serve as cladding streams. Therefore, our device can switch from the focusing mode to diverging mode. In the diverging mode, a liquid with high refractive index works as core stream, and a liquid with refractive index matching PDMS is used as cladding streams, Figure 1 (b). Owing to the higher refractive index of the core stream and the tuneable lens interface, 
the divergence of the light beam can be expanded and adjusted. The divergent beam can be used as an in-situ laser sheet for micro particle image velocimetry (microPIV) measurement. Combining the performance of focusing and diverging, our device can greatly enhance the tunability of the focal length of optofluidic lens. Moreover, the width of light beam can be tuned from approximately $100 \mu \mathrm{m}$ (focusing mode) to $1 \mathrm{~mm}$ (diverging mode). The multiple lens effects to modify the in-plane light make the bi-concave lens reported in this paper more adaptive to the lab-on-chip applications such as on-chip fluorescence detection and micro-particle manipulation [18], and flow cytometry [19].

\section{Device concept and modelling}

Figure 1 illustrates the configuration of our device. The optofluidic lens is hydrodynamically formed in a rectangular chamber measuring $1 \mathrm{~mm} \times 1 \mathrm{~mm}$, which has one core inlet, two pairs of cladding inlets and two outlets. The widths of the inlets and outlets are $50 \mu \mathrm{m}$. To characterize the performance of the concave lens by ray tracing, an optical fibre is introduced by a pre-defined channel with a width of $130 \mu \mathrm{m}$. The distance between the tip of fibre and the centre of the rectangular chamber is $4 \mathrm{~mm}$. An aperture with a size of $400 \mu \mathrm{m}$ is built by filling ink into two channels. A raytracing chamber filled with fluorescence dye is placed behind the rectangular chamber to visualize the propagation of light rays.

A two-dimensional numerical model is built based on the above geometry of the test device. The level-set method is applied to treat the interfaces between different 
fluids [20]. The level-set function is defined as

$$
\phi=\left\{\begin{array}{lr}
-d, & \vec{x} \in \Omega_{\text {cladding }} \\
0, & \vec{x} \in \Gamma \\
+d, & \vec{x} \in \Omega_{\text {core }}
\end{array}\right.
$$

where $x$ is the position vector in the computational domain $\Omega$, and $d$ is the shortest normal distance from the interface $\Gamma$. The domain of interest consists of a single special fluid of variable properties. The density $\rho$ and the dynamic viscosity $\mu$ of the special fluid are expressed as

$$
\begin{gathered}
\rho=H \rho_{\text {core }}+(1-H) \rho_{\text {cladding }} \\
\mu=H \mu_{\text {core }}+(1-H) \mu_{\text {cladding }}
\end{gathered}
$$

where the smeared Heaviside function $H$ is given by

$$
H(\phi)=\left\{\begin{array}{cr}
0, & \phi<-\varepsilon \\
\frac{\phi+\varepsilon}{2 \varepsilon}+\frac{1}{2 \pi} \sin \left(\frac{\pi \phi}{\varepsilon}\right), & |\phi| \leq \varepsilon \\
1, & \phi>+\varepsilon
\end{array}\right.
$$

The motion of this special fluid is governed by the Navier-Stokes equations, i.e.

$$
\nabla \cdot \vec{u}=0
$$

$$
\nabla \cdot(\rho \vec{u} \vec{u})=-\nabla p+\nabla \cdot\left[\mu\left(\nabla \vec{u}+\nabla \vec{u}^{T}\right)\right]
$$

The motion of the interface, i.e. the level-set function $\phi$, is given by

$$
\vec{u} \cdot \nabla \phi=0
$$


To maintain $\phi$ as a distance function, $\phi$ is set to the steady-state solution of the following equation:

$$
\frac{\partial \phi^{\prime}}{\partial \bar{t}}=\overline{s g n}(\phi)\left(1-\left|\nabla \phi^{\prime}\right|\right)
$$

where $\bar{t}$ is a pseudo-time that is employed for redistancing purpose only, and sgn is given by

$$
\overline{\operatorname{sgn}}(\phi)=\frac{\phi}{\sqrt{\phi^{2}+|\nabla \phi|^{2}(\Delta x)^{2}}}
$$

For boundary conditions, the average velocity at the inlet is set as: $u_{c}=q_{c} /(w h)$, where $q_{c}$ is the volumetric flow rate set by the syringe pump in the corresponding experiment and $w$ and $h$ are the channel width and height respectively. Outflow boundary conditions are used for the outlet. The initial level-set function is set to an assumed shape and evolved under Eq. 7 to its steady state shape.

The finite-volume method is employed to address the equation 5 and 6 [21] to solve the velocity-pressure coupling. A staggered grid is used in this computation. The scalar variables are stored at the centers of the control volume, while the velocities are located at the control volume faces. In this work, the power law of [21] is used to model the combined convection-diffusion effect in the momentum equations. The level-set and redistancing functions 7 and 8 are implemented within a narrow-band region near the interface instead of the whole computational domain to reduce computational effort. The level-set equation 2 is solved with the high order fast sweeping method $[22,23]$. The computed positions of the interface were transferred into a custom Matlab program to implement the numerical ray-tracing. 
3 Fabrication and experimental setup

The test devices were fabricated in polydimethylsiloxane (PDMS) using the standard soft lithography technique [24]. The mask was printed on a transparency film with a resolution of 8000 dpi. The transparency mask was subsequently used for defining the negative mould of the microfluidic network in a $150 \mu \mathrm{m}$ thick SU-8 layer. PDMS was mixed from the two components with a weight ratio of 10:1, then poured into the SU-8 mould. The PDMS was cured in vacuum oven at $60^{\circ} \mathrm{C}$ and cooled at room temperature for 24 hours. The PDMS was then peeled off from the master mould. Subsequently, access holes were opened by a puncher. The moulded part was subsequently bonded to another flat PDMS part after treating both surfaces with oxygen plasma. Needles with an inner diameter of $0.33 \mathrm{~mm}$ and an outer diameter $0.64 \mathrm{~mm}$ were press-fitted into the access holes and worked as fluidic interconnects. With this technology, a channel height of $150 \mu \mathrm{m}$ can be achieved for the microfluidic network of our device.

In our experiment, all liquids were kept in $5 \mathrm{ml}$ glass syringes, which are driven by syringe pumps (KDS230, KD Scientific Inc, USA) to attain the required flow rate. A sensitive CCD camera (HiSense MKII) attached to a microscope was used to capture the gray-scale images. The bright-colored field images were recorded with a digital camera (DCRDVD803E, SONY). In the focusing mode, Ethanol (RI n=1.36; viscosity $\mu=1.2 \times 10^{-3} \mathrm{Ns} / \mathrm{m}^{2}$ ) was used as the core liquid, cinnamaldehyde (RI $\mathrm{n}=1.62$; viscosity $\mu=5.7 \times 10^{-3} \mathrm{Ns} / \mathrm{m}^{2}$ ) as the cladding liquid, and a mixture of $73.5 \%$ ethylene glycol and $26.5 \%$ ethanol (viscosity $\mu=9.8 \times 10^{-3} \mathrm{Ns} / \mathrm{m}^{2}$ ) with a refractive index matching PDMS ( $\mathrm{n}=1.412)$ worked as the auxiliary cladding liquid. During the test, the flow rate of the auxiliary cladding stream was fixed at one-tenth that of the cladding stream. In the diverging mode, the pumping of core liquid was stopped to enable the 
liquid from the cladding inlets to converge into a new core stream (cinnamaldehyde), and therefore changing the role of the auxiliary cladding liquid from light-scattering protection to providing a mis-match of refractive index with that of the core stream to refract the light rays. Laser light (output $=200 \mathrm{~mW}$, wavelength $\lambda=532 \mathrm{~nm}$ ) was coupled into a multimode optical fibre (AFS105/125Y, THORLABS Inc.) with a numerical aperture $\mathrm{NA}=0.22$, which was inserted into a pre-fabricated microchannel with a width of $130 \mu \mathrm{m}$ to act as a point light source. The light rays refracted by the concave lens are visualized in a ray-tracing chamber filled with fluorescence dye Rhodamine B (Sigma-Aldrich, excitation wavelength of $540 \mathrm{~nm}$, emission wavelength of $625 \mathrm{~nm}$ ).

\section{Results and discussion}

Figure 2 shows the manipulation of the lens interface by adjusting the flow rate ratio between core and cladding streams for the focusing mode of the optofluidic bi-concave lens. The flow rate provided by the syringe pump is not smooth causing fluctuation of the lens interfaces. The error bars in Figures 2, 4, 6 and 8 reflect the effect of flow fluctuation inside the lens chambers. During the test, the flow rate of the cladding streams and the auxiliary cladding stream were fixed at $1 \mathrm{~mL} / \mathrm{h}$ and $0.1 \mathrm{~mL} / \mathrm{h}$, respectively. The flow rate of the core stream was increased from $2 \mathrm{~mL} / \mathrm{h}$ to $14 \mathrm{~mL} / \mathrm{h}$. At low

flow rate ratio $\left(\phi_{\text {core }} / \phi_{\text {cladding }}\right)$, the curvature of the interface between the core and cladding streams is small due to the relatively straight streamlines in the centre part of the rectangular chamber. When the interface approaches the side-wall of the chamber at high flow rate ratio, the value of the curvature decreases owing to the less curved streamlines near the boundary. When the position of the interface locates between the centre and side-wall of the chamber, the curvature can achieve a maximum value. As 
a result of the significantly lower flow rate of the auxiliary cladding stream compared to the cladding streams, a thin layer liquid with a refractive index matching that of PDMS forms on the side-wall of the chamber. This thin layer prevents the scattering of the incident light on the concave lens that is due to the roughness of the PDMS surface, and the small thickness of the layer is supposed to reduce the refraction effect by this layer itself.

Ray tracing method was carried out experimentally and numerically to investigate the optical performance of the optofluidic concave lens in the focusing mode. In the numerical simulation, the shape of the interface between different streams was computed with level-set method and imported into a custom Matlab program which implements the ray tracing based on Snell's law. In the experiment, a green laser with a wavelength of $\lambda=532 \mathrm{~nm}$ was used to excite the fluorescence dye in the ray-tracing chamber, and the visualized light rays were recorded in gray-scale images. Due to the refractive index of the core liquid (ethanol $n=1.36$ ) being lower than that of the cladding liquid (cinnamaldehyde $\mathrm{n}=1.62$ ), the optofluidic concave lens possesses light focusing effect. The simulation and experimental results, which were obtained at flow rate ratio of 2 , 6 , and 10, are compared and shown in Figure 3. At the low flow rate ratio $\phi=2$, the concave lens has a longer focal length owing to the small curvature when the interface is positioned around the centre part of the chamber, Fig. 3(a). By increasing the flow rate ratio, the interface becomes more curved, which results in a shorter focal length, Fig. 3(b). When the interface approaches the side-wall of the rectangular chamber, the curvature of the interface decreases, thus elongating the focal length, Fig. 3(c).

To measure the focal length of the optofluidic concave lens, the gray-scale images of the light rays in the ray-tracing chamber were captured, and rays with the angular aperture in the image space were extracted to calculate the intersection position of the 
rays and the optical axis to obtain the image distance. The focal length of the concave lens at each corresponding flow rate ratio can be calculated by applying the conjugate relationship between the object and the image. The experimental results are shown and compared with our numerical analysis in Fig. 4.

To demonstrate the importance of the auxiliary cladding stream in the focusing mode of the optofluidic bi-concave lens, we compare the optical performance of the concave lens with and without this stream. We carried out the testing at a flow rate ratio of 6 . The ray-tracing images were captured and shown in Fig. 5 (a) and (b). The intensity distributions along the focal plane were extracted and compared in Fig. 5 (c). The comparison shows that the concave lens with auxiliary cladding stream has a narrower beam width at the focal plane, and its intensity profile is more smoothly and tightly distributed. However, the beam width of the concave lens without the auxiliary cladding stream is wider, and the intensity profile is not continuously distributed. This phenomenon is due to the rough PDMS surface, which would diffusively refract the light rays when the refractive index of cladding stream is different from that of PDMS.

The focusing mode can be simply converted to diverging mode by stopping the pumping of liquid (ethanol) from the core inlet. By this way, the previous cladding liquid (cinnamaldehyde) in focusing mode will converge in the rectangular chamber and become a core stream in the diverging mode, and the role of the auxiliary cladding liquid (mixture of ethylene glycol and ethanol) changes from preventing light-scattering to refracting light rays (Fig. 1 (c)). Therefore, the core and cladding liquids involved in the diverging mode testing are cinnamaldehyde and the mixture of $73.5 \%$ ethylene glycol and $26.5 \%$ ethanol respectively. Figure 6 shows the relationship between the flow rate ratio $\left(\phi_{\text {core }} / \phi_{\text {cladding }}\right)$ and the curvature of the interface for the diverging mode. In the test, the core stream was pumped with a fixed flow rate $2 \mathrm{~mL} / \mathrm{h}$, and the flow 
rate of cladding stream was varied from $0.2 \mathrm{~mL} / \mathrm{h}$ to $5 \mathrm{~mL} / \mathrm{h}$. Because the streamlines at the centre and near the side-wall of the rectangular chamber have relatively straight line shape, the values of the curvature of the interface at low and high flow rate ratios are smaller. And when the interface is positioned between the centre and the side-wall of the chamber, the curvature can achieve a maximum value. This trend of the curve is similar to that in the focusing mode testing.

Due to the higher refractive index of the core liquid in the diverging mode, the optofluidic bi-concave lens spreads the light rays thus expanding the divergent angle of the light beam originating from the optical fiber. To investigate the optical performance of this concave lens, we have also implemented experimental and numerical ray-tracing method in the same way as the investigation of the focusing mode. The comparison between experimental results and numerical simulation is illustrated in Figure 7. The results were obtained at flow rate ratios of 20,10,2 and 0.5. At the highest flow rate ratio of 20 , the interface is close to the side-wall of the chamber resulting in the small curvature of the interface having little impact on the refraction of light rays. Therefore the divergent angle of the light beam from the fiber remains almost unchanged after passing the concave lens. With a decrease in the flow rate ratio, the curvature of the interface is increased, so the concave lens greatly expands the divergent angle of the light beam. However, as the interface approaches the centre of the chamber, its curvature drops, and the divergent angle of the light beam reduces as well. The divergent angle at each corresponding flow rate ratio was measured and compared with the numerical analysis in Fig. 8. In the measurement, the experimental ray-tracing results were recorded in gray-scale images, and the rays with the angular aperture in the image space were extracted. The included angle of the rays is defined as the divergent angle. Owing to the aperture, the opening angle of the light beam originating 
from the fiber is 6 degree before the refraction by the concave lens. After passing the lens, the divergent angle can be expanded to more than 20 degree. Since this tunable expansion of the divergent angle can help to control the radiant flux and the size of the illumination area, it may make the way of fluorescence excitation more flexible. Moreover, this tunable diverging lens may be very useful to correct spherical aberration in an optofluidic lens system.

\section{Conclusions}

In this paper, we reported the design and characterization of an optofluidic bi-concave lens, which can perform both light focusing and diverging. The new design was modelled numerically. Level set method was applied to compute the fluid dynamics and capture the fluid interface. The computation results of interface positions were transferred to Matlab, where numerical ray tracing was carried out by using Snell's law based on the interface positions. Experiment was conducted to verify our modelling. And the experimental results agree well with the numerical analysis, which strengthens the validity of our method of numerical simulation. Therefore this numerical method can be used to analyze similar design of optofluidics in the future with good reliability. In the focusing mode of the concave lens, a liquid (ethanol) with low refractive index is used as the core stream and a liquid (cinnamaldehyde) with higher refractive index serves as cladding stream. The relatively lower refractive index of the core and the concave shape of the interface conjunctly enable the lens to focus light. An auxiliary cladding stream with low flow rate was introduced to effectively minimize the light scattering at the rough surface of the chamber wall. This concept of auxiliary cladding stream can be further applied to other optofluidic designs, in which the light may scatter at the 
optically-rough contact surface between refractive index mis-matching substances. The focusing mode of the optofluidic bi-concave lens can be simply switched to diverging mode by stopping pumping the liquid from core inlet. The previous cladding liquid (cinnamaldehyde) would join together in the chamber and become the core stream for the diverging mode. The auxiliary cladding liquid would change its function from avoiding light-scattering to becoming the cladding stream for the diverging mode. Combining the performance of focusing and diverging, the tunability of the focal length of the optofluidic lens and the width of the light beam can be greatly enhanced. This improvement of tunability will make this optofluidic bi-concave lens more adaptive to the lab-on-chip applications.

\section{References}

1. Chabinyc, M.L., D.T. Chiu, J.C. McDonald, A.D. Stroock, J.F. Christian, A.M. Karger, and G.M. Whitesides, An integrated fluorescence detection system in poly(dimethylsiloxane) for microfluidic applications. Analytical Chemistry, 73, 4491-4498 (2001).

2. Fonseca, A., I.M. Raimundo Jr, J.J.R. Rohwedder, R.S. Lima, and M.C.U. Araujo, A microfluidic device with integrated fluorimetric detection for flow injection analysis. Analytical and Bioanalytical Chemistry, 396, 715-723 (2009).

3. Kuhn, S., B.S. Phillips, E.J. Lunt, A.R. Hawkins, and H. Schmidt, Ultralow power trapping and fluorescence detection of single particles on an optofluidic chip. Lab on a Chip Miniaturisation for Chemistry and Biology, 10, 189-194 (2010).

4. Qi, S., X. Liu, S. Ford, J. Barrows, G. Thomas, K. Kelly, A. McCandless, K. Lian, J. Goettert, and S.A. Soper, Microfluidic devices fabricated in poly(methyl methacrylate) using hot-embossing with integrated sampling capillary and fiber optics for fluorescence detection. Lab on a Chip - Miniaturisation for Chemistry and Biology, 2, 88-95 (2002).

5. Tung, Y.C., M. Zhang, C.T. Lin, K. Kurabayashi, and S.J. Skerlos, PDMS-based optofluidic micro flow cytometer with two-color, multi-angle fluorescence detection capability using PIN photodiodes. Sensors and Actuators, B: Chemical, 98, 356-367 (2004). 
6. Camou, S., H. Fujita, and T. Fujii, PDMS 2D optical lens integrated with microfluidic channels: Principle and characterization. Lab on a Chip - Miniaturisation for Chemistry and Biology, 3, 40-45 (2003).

7. Seo, J. and L.P. Lee, Disposable integrated microfluidics with self-aligned planar microlenses. Sensors and Actuators, B: Chemical, 99, 615-622 (2004).

8. Mao, X., J.R. Waldeisen, B.K. Juluri, and T.J. Huang, Hydrodynamically tunable optofluidic cylindrical microlens. Lab on a Chip - Miniaturisation for Chemistry and Biology, 7, 1303-1308 (2007).

9. Rosenauer, M. and M.J. Vellekoop, 3D fluidic lens shaping - A multiconvex hydrodynamically adjustable optofluidic microlens. Lab on a Chip - Miniaturisation for Chemistry and Biology, 9, 1040-1042 (2009)

10. Song, C., N.T. Nguyen, S.H. Tan, and A.K. Asundi, A tuneable micro-optofluidic biconvex lens with mathematically predictable focal length. Microfluidics and Nanofluidics, 9, 889-896 (2010).

11. Song, C., N.T. Nguyen, S.H. Tan, and A.K. Asundi, Modelling and optimization of micro optofluidic lenses. Lab on a Chip - Miniaturisation for Chemistry and Biology, 9, 1178-1184 (2009)

12. Tang, S.K.Y., C.A. Stan, and G.M. Whitesides, Dynamically reconfigurable liquid-core liquid-cladding lens in a microfluidic channel. Lab on a Chip - Miniaturisation for Chemistry and Biology, 8, 395-401 (2008).

13. Shi, J., Z. Stratton, S.C.S. Lin, H. Huang, and T.J. Huang, Tunable optofluidic microlens through active pressure control of an air-liquid interface. Microfluidics and Nanofluidics, 1-6 (2009).

14. Mao, X., S.C.S. Lin, M.I. Lapsley, J. Shi, B.K. Juluri, and T.J. Huang, Tunable Liquid Gradient Refractive Index (L-GRIN) lens with two degrees of freedom. Lab on a Chip Miniaturisation for Chemistry and Biology, 9, 2050-2058 (2009).

15. Song, C., N.T. Nguyen, A.K. Asundi, and S.H. Tan, Tunable micro-optofluidic prism based on liquid-core liquid-cladding configuration. Optics Letters, 35, 327-329 (2010).

16. Psaltis, D., S.R. Quake, and C. Yang, Developing optofluidic technology through the fusion of microfluidics and optics. Nature, 442, 381-386 (2006). 
17. Song, C., N.T. Nguyen, A.K. Asundi, and C.L.N. Low, Biconcave micro-optofluidic lens with low-refractive-index liquids. Optics Letters, 34, 3622-3624 (2009).

18. Yin, D., E.J. Lunt, M.I. Rudenko, D.W. Deamer, A.R. Hawkins, and H. Schmidt, Planar optofluidic chip for single particle detection, manipulation, and analysis. Lab on a Chip Miniaturisation for Chemistry and Biology, 7 1171-1175 (2007).

19. Godin, J., V. Lien, and Y.H. Lo, Demonstration of two-dimensional fluidic lens for integration into microfluidic flow cytometers. Applied Physics Letters, 89 (2006).

20. Osher, S. and J.A. Sethian, Fronts propagating with curvature-dependent speed: Algorithms based on Hamilton-Jacobi formulations. Journal of Computational Physics, 79 12-49 (1988).

21. Patankar, J. U., Numerical Heat Transfer and Fluid Flow, Hemisphere, New York, (1980).

22. Peng, D., B. Merriman, S. Osher, H. Zhao, and M. Kang, A PDE-Based Fast Local Level Set Method. Journal of Computational Physics, 155 410-438 (1999).

23. Zhang, Y.T., H.K. Zhao, and J. Qian, High order fast sweeping methods for static Hamilton-Jacobi equations. Journal of Scientific Computing, 29, 25-56 (2006).

24. Xia, Y., and G. M. Whitesides, Soft lithography. Annual Review of Material Science, 28, 153-184 (1998). 


\section{List of Figures}

- Figure 1: Schematics: (a) Illustration of the optofluidic concave lens in the focusing mode; (b) Bright field image of the concave lens focusing a light beam. The liquids for the core, cladding, and auxiliary cladding streams are ethanol, cinnamaldehyde, and the mixture of $73.5 \%$ ethylene glycol and $26.5 \%$ ethanol respectively. The flow rate ratio between core and cladding stream is 6 . The flow rate of the auxiliary cladding stream is set as one-tenth of that of the cladding stream; (c) Illustration of the concave lens in the diverging mode; (d) Bright field image of the concave lens spreading a light beam. The liquids for the core and cladding streams are cinnamaldehyde and the mixture of $73.5 \%$ ethylene glycol and $26.5 \%$ ethanol respectively. The flow rate ratio between core and cladding stream is 2.8 .

- Figure 2:Relationship between the flow rate ratio and the curvature of the interface in the focusing mode of the optofluidic bi-concave lens. Ethanol (RI $\mathrm{n}=1.36$; viscosity $\mu=1.2 \times 10^{-3} \mathrm{Ns} / \mathrm{m}^{2}$ ) is used as the core liquid, cinnamaldehyde (RI $\mathrm{n}=1.62$; viscosity $\mu=5.7 \times 10^{-3} \mathrm{Ns} / \mathrm{m}^{2}$ ) serves as cladding liquid, and a mixture of $73.5 \%$ ethylene glycol and $26.5 \%$ ethanol (viscosity $\mu=9.8 \times 10^{-3} \mathrm{Ns} / \mathrm{m}^{2}$ ) with a refractive index matching PDMS ( $\mathrm{n}=1.412)$ works as auxiliary cladding liquid. The flow rate of the cladding streams and the auxiliary cladding stream were fixed at 1 $\mathrm{mL} / \mathrm{h}$ and $0.1 \mathrm{~mL} / \mathrm{h}$, respectively. The flow rate of the core stream was increased from $2 \mathrm{~mL} / \mathrm{h}$ to $14 \mathrm{~mL} / \mathrm{h}$. The thin layer close to the side-wall of the chamber in the inset is the auxiliary cladding stream.

- Figure 3:Comparisons between experimental and numerical ray-tracing results under conditions of different flow rate ratios between core and cladding streams in the focusing mode. The shape of the interfaces between different streams involved 
in the numerical simulation are computed with level-set method and imported into Matlab environment for ray-tracing. The experimental results were retrieved by using an optical fibre to introduce an incident green laser light (wavelength $\lambda=532 \mathrm{~nm})$ on the concave lens. The refracted light rays were visualized by the fluorescence dye in the ray-tracing chamber and recorded by CCD camera in grayscale images.

- Figure 4:Relationship between the focal length of the optofluidic bi-concave lens in the focusing mode and the flow rate ratio between core and cladding streams. The core liquid is ethanol, cladding liquid is cinnamaldehyde, and the auxiliary cladding liquid is the mixture of $73.5 \%$ ethylene glycol and $26.5 \%$ ethanol. The flow rates of the cladding and auxiliary cladding streams were fixed at $1 \mathrm{~mL} / \mathrm{h}$ and $0.1 \mathrm{~mL} / \mathrm{h}$ respectively, while adjusting the flow rate of core stream to tune the focal length.

- Figure 5:Comparison between the optofluidic bi-concave lens with and without the auxiliary cladding streams in the focusing mode. The light beam is focused under a condition of flow rate ratio 6. (a) A well focused light beam by a concave lens with the auxiliary cladding stream; (b) a focused and scattered light beam by a concave lens without the auxiliary cladding stream; (c) intensity profiles extracted from the gray-scale images (a) and (b).

- Figure 6:Relationship between the flow rate ratio and the curvature of the interface in the diverging mode of the optofluidic bi-concave lens. Cinnamaldehyde (RI $n=$ 1.62 ; viscosity $\mu=5.7 \times 10^{-3} \mathrm{Ns} / \mathrm{m}^{2}$ ) serves as core liquid, and a mixture of $73.5 \%$ ethylene glycol and $26.5 \%$ ethanol (viscosity $\mu=9.8 \times 10^{-3} \mathrm{Ns} / \mathrm{m}^{2}$ ) with a refractive index matching PDMS $(\mathrm{n}=1.412)$ works as cladding liquid. The flow 
rate of the core stream was fixed at $2 \mathrm{~mL} / \mathrm{h}$. The flow rate of the cladding stream was tuned from $0.2 \mathrm{~mL} / \mathrm{h}$ to $5 \mathrm{~mL} / \mathrm{h}$.

- Figure 7:Comparisons between experimental and numerical ray-tracing results under conditions of different flow rate ratios between core and cladding streams in the diverging mode. The shape of the interfaces between different streams involved in the numerical simulation are computed with level-set method and imported in Matlab environment for ray-tracing. The experimental results were retrieved by using an optical fibre to introduce an incident green laser light (wavelength $\lambda=532$ $\mathrm{nm}$ ) on the concave lens. The refracted light rays were visualized by the fluorescence dye in the ray-tracing chamber and recorded by CCD camera in gray-scale images.

- Figure 8:Relationship between the divergent angle of light beam and the flow rate ratio between core and cladding streams in the diverging mode. The core liquid is cinnamaldehyde, and the cladding liquid is the mixture of $73.5 \%$ ethylene glycol and $26.5 \%$ ethanol. The flow rates of the core streams were fixed at $2 \mathrm{~mL} / \mathrm{h}$, while adjusting the flow rate of cladding stream to tune the divergent angle. 


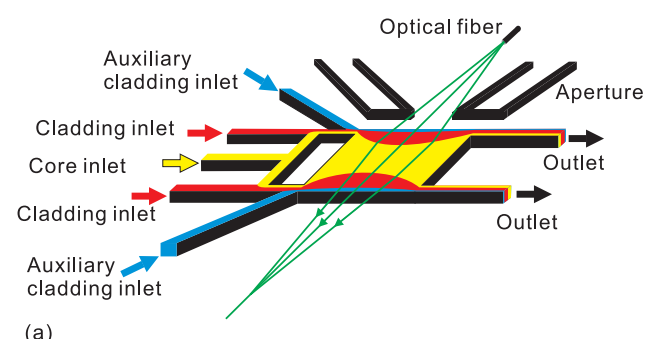

(a)
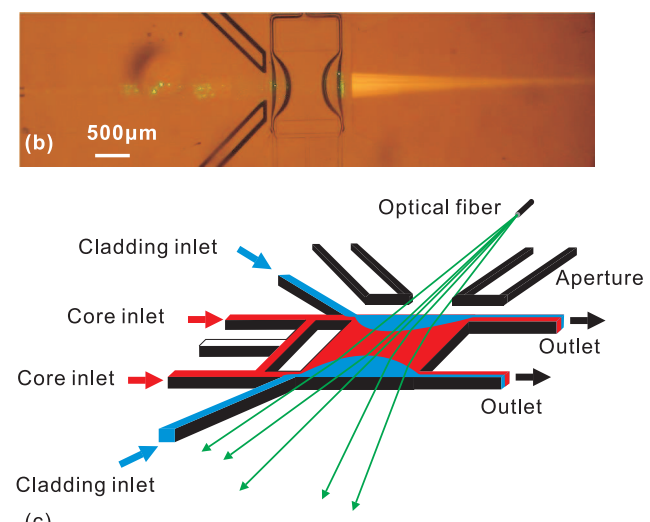

(c)

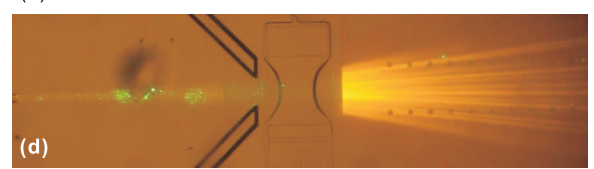

Fig. 1 Schematics: (a) Illustration of the optofluidic concave lens in the focusing mode; (b) Bright field image of the concave lens focusing a light beam. The liquids for the core, cladding, and auxiliary cladding streams are ethanol, cinnamaldehyde, and the mixture of $73.5 \%$ ethylene glycol and $26.5 \%$ ethanol respectively. The flow rate ratio between core and cladding stream is 6 . The flow rate of the auxiliary cladding stream is set as one-tenth of that of the cladding stream; (c) Illustration of the concave lens in the diverging mode; (d) Bright field image of the concave lens spreading a light beam. The liquids for the core and cladding streams are cinnamaldehyde and the mixture of $73.5 \%$ ethylene glycol and $26.5 \%$ ethanol respectively. The flow rate ratio between core and cladding stream is 2.8 . 


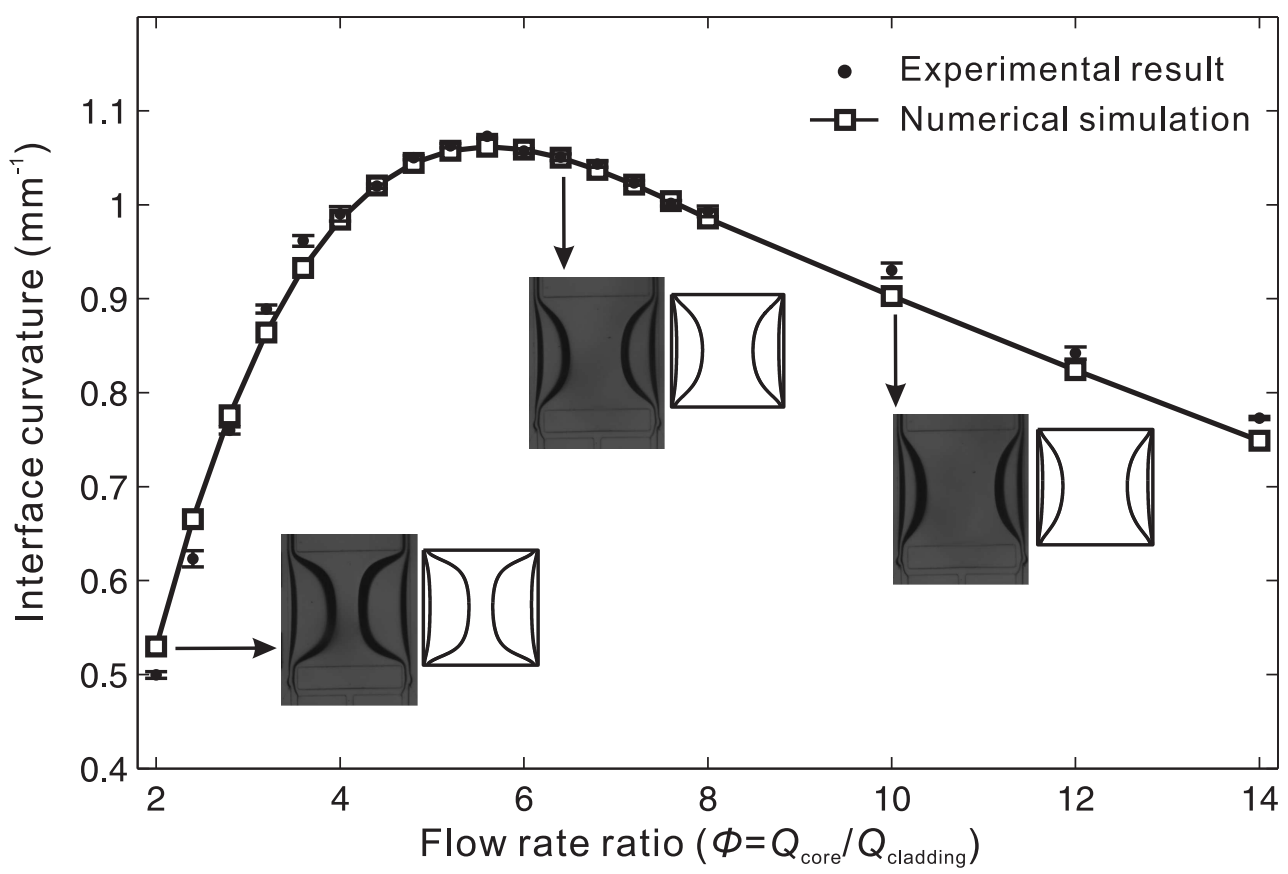

Fig. 2 Relationship between the flow rate ratio and the curvature of the interface in the focusing mode of the optofluidic bi-concave lens. Ethanol (RI $\mathrm{n}=1.36$; viscosity $\mu=1.2 \times$ $10^{-3} \mathrm{Ns} / \mathrm{m}^{2}$ ) is used as the core liquid, cinnamaldehyde (RI $\mathrm{n}=1.62$; viscosity $\mu=5.7 \times$ $10^{-3} \mathrm{Ns} / \mathrm{m}^{2}$ ) serves as cladding liquid, and a mixture of $73.5 \%$ ethylene glycol and $26.5 \%$ ethanol (viscosity $\mu=9.8 \times 10^{-3} \mathrm{Ns} / \mathrm{m}^{2}$ ) with a refractive index matching PDMS ( $\mathrm{n}=1.412$ ) works as auxiliary cladding liquid. The flow rate of the cladding streams and the auxiliary cladding stream were fixed at $1 \mathrm{~mL} / \mathrm{h}$ and $0.1 \mathrm{~mL} / \mathrm{h}$, respectively. The flow rate of the core stream was increased from $2 \mathrm{~mL} / \mathrm{h}$ to $14 \mathrm{~mL} / \mathrm{h}$. The thin layer close to the side-wall of the chamber in the inset is the auxiliary cladding stream. 


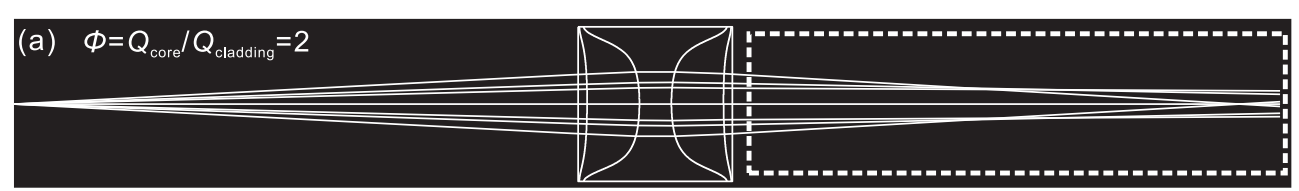

\section{Light propagation direction}
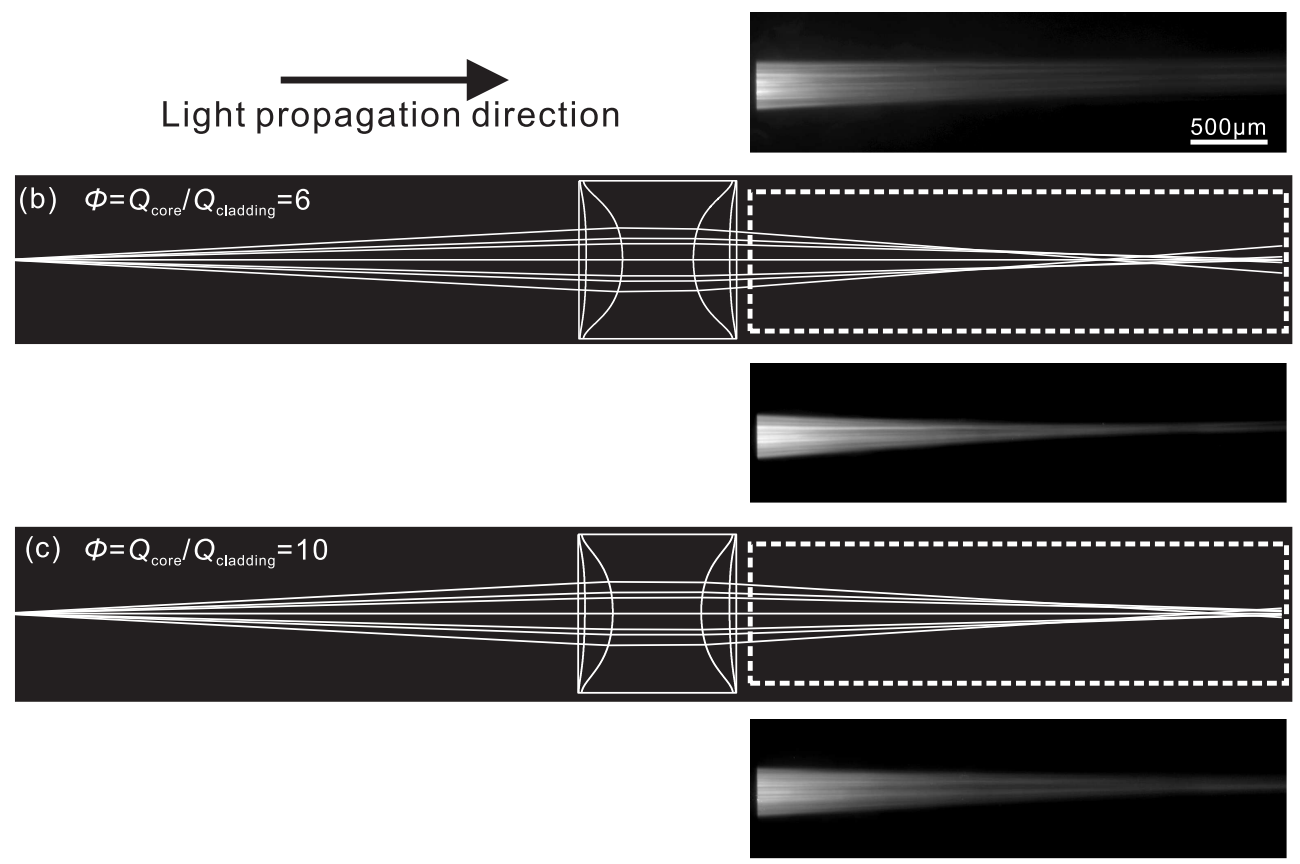

Fig. 3 Comparisons between experimental and numerical ray-tracing results under conditions of different flow rate ratios between core and cladding streams in the focusing mode. The shape of the interfaces between different streams involved in the numerical simulation are computed with level-set method and imported into Matlab environment for ray-tracing. The experimental results were retrieved by using an optical fibre to introduce an incident green laser light (wavelength $\lambda=532 \mathrm{~nm}$ ) on the concave lens. The refracted light rays were visualized by the fluorescence dye in the ray-tracing chamber and recorded by CCD camera in gray-scale images. 


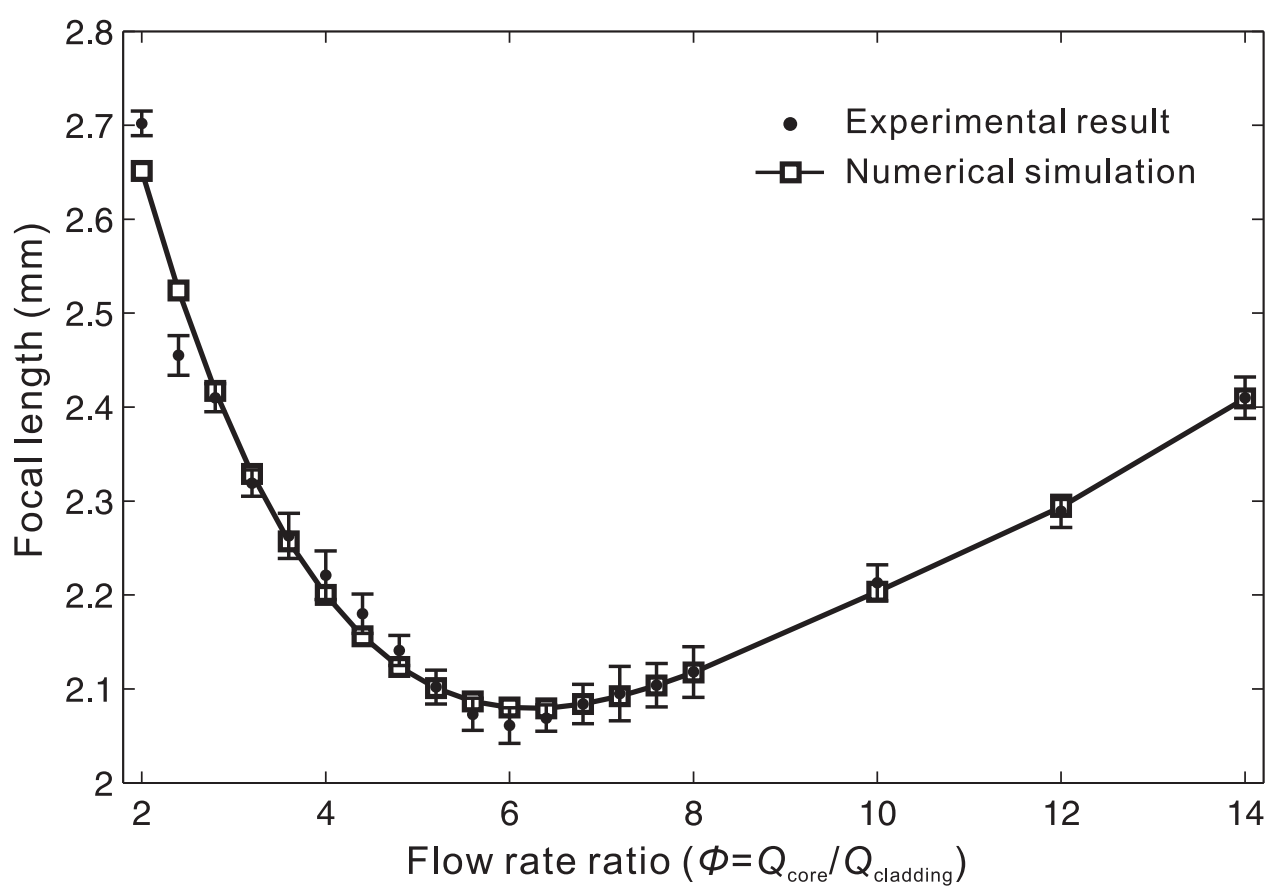

Fig. 4 Relationship between the focal length of the optofluidic bi-concave lens in the focusing mode and the flow rate ratio between core and cladding streams. The core liquid is ethanol, cladding liquid is cinnamaldehyde, and the auxiliary cladding liquid is the mixture of $73.5 \%$ ethylene glycol and $26.5 \%$ ethanol. The flow rates of the cladding and auxiliary cladding streams were fixed at $1 \mathrm{~mL} / \mathrm{h}$ and $0.1 \mathrm{~mL} / \mathrm{h}$ respectively, while adjusting the flow rate of core stream to tune the focal length. 
(a)

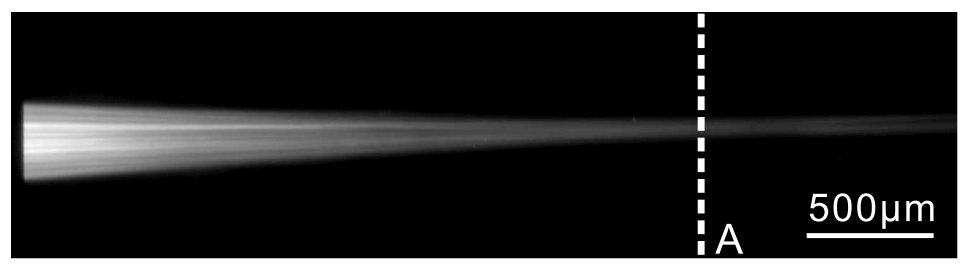

(b)
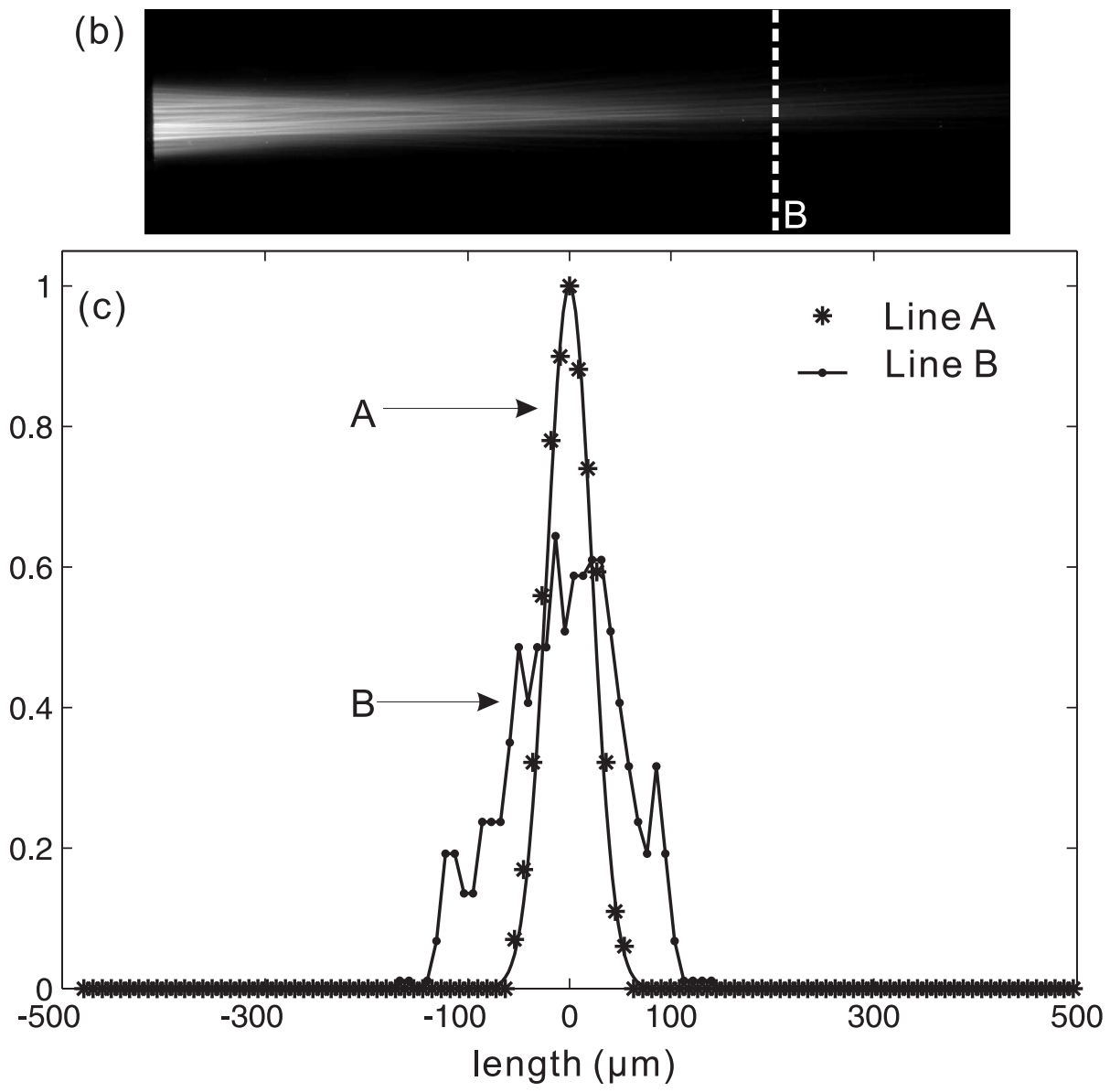

Fig. 5 Comparison between the optofluidic bi-concave lens with and without the auxiliary cladding streams in the focusing mode. The light beam is focused under a condition of flow rate ratio 6. (a) A well focused light beam by a concave lens with the auxiliary cladding stream; (b) a focused and scattered light beam by a concave lens without the auxiliary cladding stream; (c) intensity profiles extracted from the gray-scale images (a) and (b). 


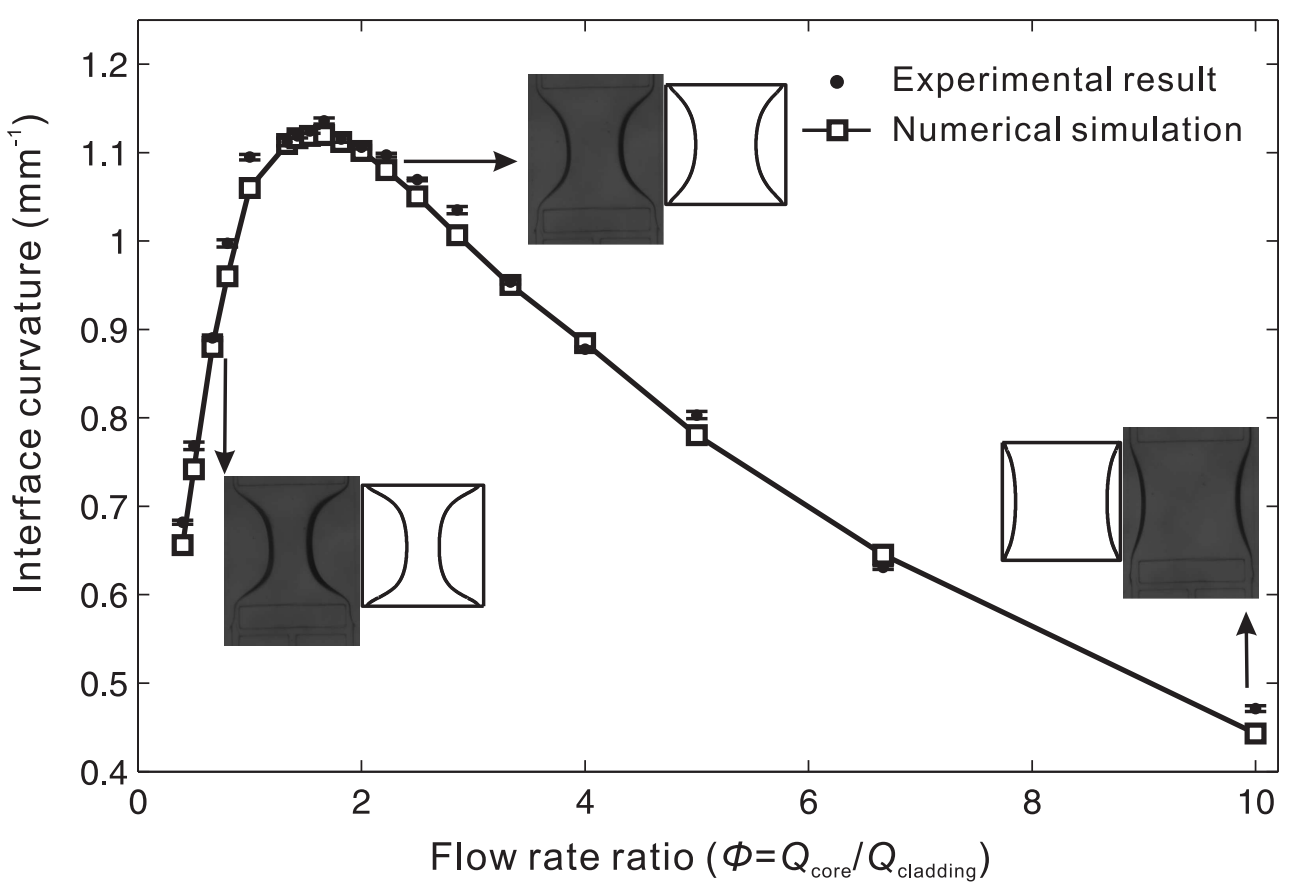

Fig. 6 Relationship between the flow rate ratio and the curvature of the interface in the diverging mode of the optofluidic bi-concave lens. Cinnamaldehyde (RI $\mathrm{n}=1.62$; viscosity $\mu=5.7 \times 10^{-3} \mathrm{Ns} / \mathrm{m}^{2}$ ) serves as core liquid, and a mixture of $73.5 \%$ ethylene glycol and $26.5 \%$ ethanol (viscosity $\mu=9.8 \times 10^{-3} \mathrm{Ns} / \mathrm{m}^{2}$ ) with a refractive index matching PDMS (n $=1.412$ ) works as cladding liquid. The flow rate of the core stream was fixed at $2 \mathrm{~mL} / \mathrm{h}$. The flow rate of the cladding stream was tuned from $0.2 \mathrm{~mL} / \mathrm{h}$ to $5 \mathrm{~mL} / \mathrm{h}$. 

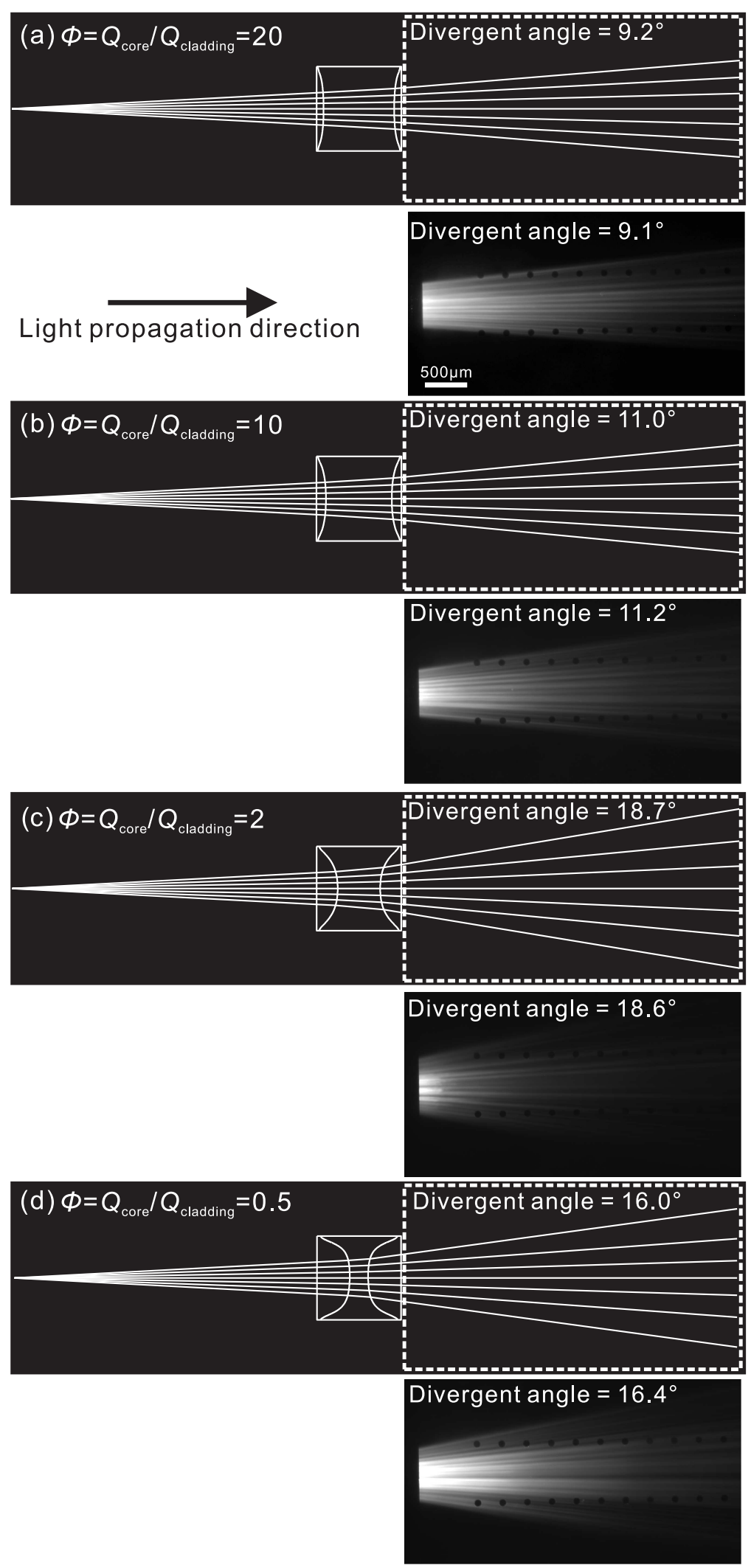

Fig. 7 Comparisons between experimental and numerical ray-tracing results under conditions of different flow rate ratios between core and cladding streams in the diverging mode. The shape of the interfaces between different streams involved in the numerical simulation are computed with level-set method and imported in Matlab environment for ray-tracing. The experimental results were retrieved by using an optical fibre to introduce an incident green laser light (wavelength $\lambda=532 \mathrm{~nm}$ ) on the concave lens. The refracted light rays were visualized 


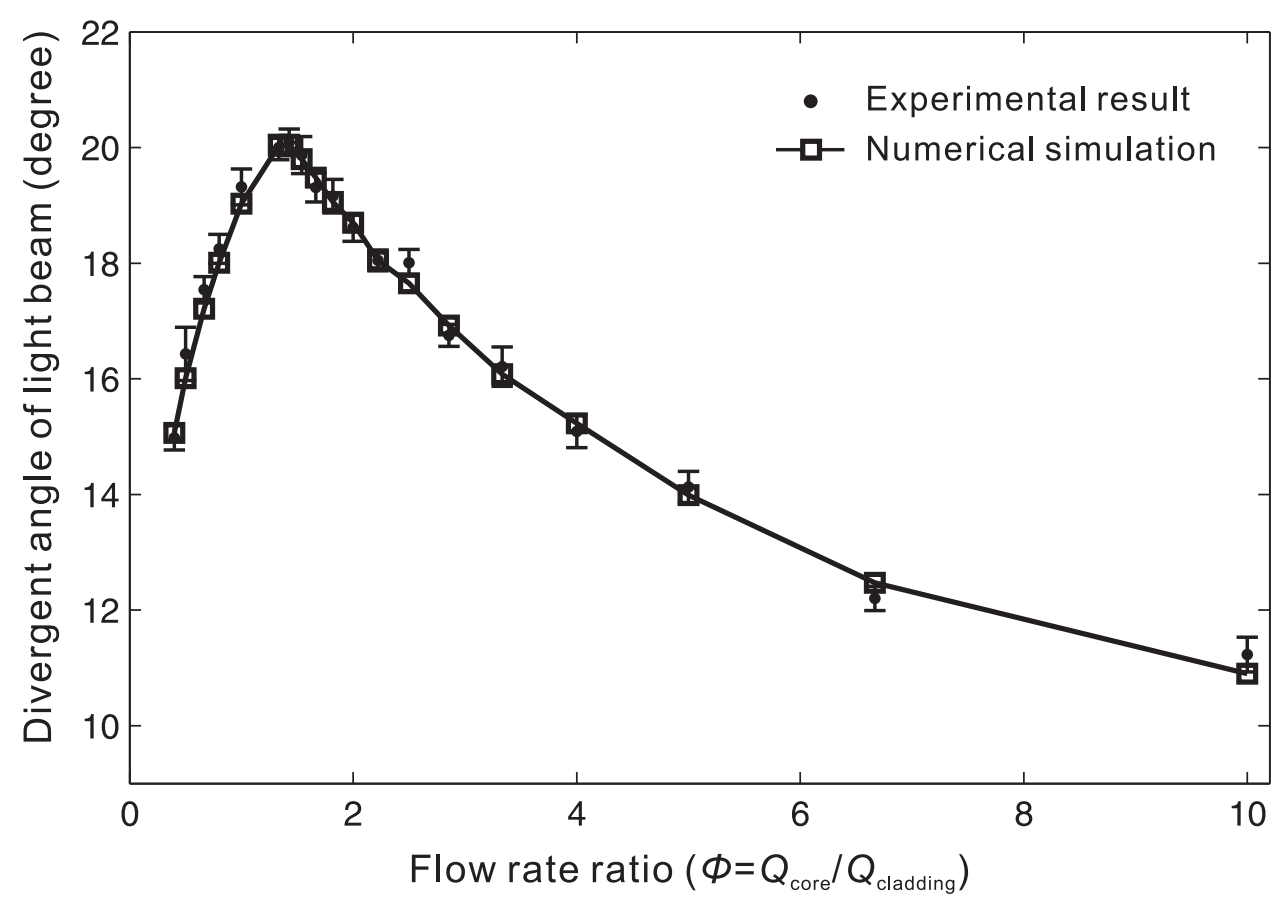

Fig. 8 Relationship between the divergent angle of light beam and the flow rate ratio between core and cladding streams in the diverging mode. The core liquid is cinnamaldehyde, and the cladding liquid is the mixture of $73.5 \%$ ethylene glycol and $26.5 \%$ ethanol. The flow rates of the core streams were fixed at $2 \mathrm{~mL} / \mathrm{h}$, while adjusting the flow rate of cladding stream to tune the divergent angle. 